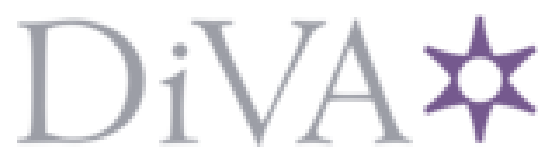

http://www.diva-portal.org

This is the published version of a paper published in Comptes rendus de l'Académie des sciences. Série 1, Mathématique.

Citation for the original published paper (version of record):

Hedenmalm, H., Jakobsson, S., Shimorin, S. (1999)

A maximum principle a la Hadamard for biharmonic operators with applications to the Bergman spaces.

Comptes rendus de l'Académie des sciences. Série 1, Mathématique, 328: 973-978

Access to the published version may require subscription.

N.B. When citing this work, cite the original published paper.

Permanent link to this version:

http://urn.kb.se/resolve?urn=urn:nbn:se:kth:diva- 182396 


\section{A maximum principle à la Hadamard for biharmonic operators with applications to the Bergman spaces}

\section{Håkan HEDENMALM, Stefan JAKOBSSON, Sergei SHIMORIN}

Department of Mathematics, University of Lund, Box 118, 22100 Lund, Sweden

E-mail: \{haakan,stefanj,shimorin $\} @$ maths.lth.se

(Reçu le 23 février 1999, accepté le 8 mars 1999)

Abstract. $\quad$ Let $\mathbb{D}$ be the open unit disk and $\Sigma$ area measure, normalized so that $\mathbb{D}$ has mass 1 . Suppose the weight $\omega: \mathbb{D} \rightarrow[0,+\infty[$ has $\operatorname{lng} \omega$ suhharmonic, and furthermore, that it has the reproducing property:

$$
h(0)=\int_{D} h(z) \omega(z) \mathrm{d} \Sigma(z)
$$

for all bounded harmonic functions $h$ on $\mathrm{D}$. Let $\Gamma_{\omega}$ be the Green function for the weighted biharmonic operator $\Delta \omega^{-1} \Delta$ on $\mathbb{D}$ with vanishing Dirichlet boundary data. We prove that $0 \leq \Gamma_{\omega}$ holds on $\mathbb{D} \times \mathbb{D}$. This result has interesting applications to the operator theory of the Bergman spaces. (C) Académie des Sciences/Elsevier, Paris

\section{Un principe du maximum à la Hadamard pour les opérateurs biharmoniques avec des applications aux espaces de Bergman}

Résumé. $\quad$ Soient $\mathbb{D}$ le disque unité ouvert et $\Sigma$ la mesure de Lebesgue dans le plan, normalisée telle que $\mathbb{D}$ soit de mesure 1 . On suppose que le poids $\omega: \mathbb{D} \rightarrow[0,+\infty[$ est tel que $\log \omega$ est sous-harmonique et que $\omega$ possède la proprieté de reproduction :

$$
h(0)=\int_{D} h(z) \omega(z) \mathrm{d} \Sigma(z)
$$

pour chaque fonction h bornée et harmonique dans $\mathbb{D}$. Soit $\Gamma_{\omega}$ la fonction de Green pour l'opérateur biharmonique pondéré $\Delta \omega^{-1} \Delta$ dans $\mathbb{D}$ pour le problème de Dirichlet. Nous montrons que $0 \leq \Gamma_{\omega}$ dans $\mathbb{D} \times \mathbb{D}$. Ce résultat a des application intéressantes à la théorie des opérateurs dans les espaces de Bergman. (C) Académie des Sciences/Elsevier, Paris

Note présentée par Jean-Pierre KaHanf. 


\section{H. Hedenmalm et al.}

\section{Version française abrégée}

On considère l'opérateur bilaplacien $\Delta^{2}$ dans le plan complexe $\mathbb{C}$, et son extension aux surfaces riemanniennes feuilletées sur le plan complexe de manière convenable. Soit $R$ une telle surface riemannienne qui, de plus, est conformément équivalente au disque unité $\mathbb{D}$. On prouve que la fonction biharmonique de Green est positive pourvu que $R$ vérifie la propriété de reproduction :

$$
h(0)=\int_{R} h(z) \mathrm{d} \Sigma(z)
$$

pour chaque fonction $h$ bornée et harmonique dans $R$ (où $\mathrm{d} \Sigma(z)=\pi^{-1} \mathrm{~d} x \mathrm{~d} y, z=x+i y$, est la mesure de Lebesgue dans le plan - normalisée comme avant - que l'on étend à chaque feuille de $R$ ). Ceci correspond à un certain principe du maximum pour les fonctions sous-biharmoniques conjecturé par Hadamard sous une hypothèse différente (à savoir, $R$ est un sous-domaine convexe de $\mathbb{C}$ ). Néanmoins, il a été démontré que l'hypothèse d'Hadamard seule n'est pas suffisante pour la validité de ce principe. L'action de l'opérateur différentiel $\Delta^{2}$ sur $R$ correspond à l'action de l'opérateur $\Delta\left|\phi^{\prime}\right|^{-2} \Delta$ sur $\mathbb{D}$ moyennant une certaine transformation conforme $\phi: \mathbb{D} \rightarrow R$. Plus généralement, on considère les opérateurs biharmoniques pondérés $\Delta \omega^{-1} \Delta$ agissant sur $\mathbb{D}$, où le poids $\omega$ est tel que $\log \omega$ est sous-harmonique, et satisfait la propriété de reproduction :

$$
h(0)=\int_{\mathbb{D}} h(z) \omega(z) \mathrm{d} \Sigma(z)
$$

pour chaque fonction $h$ bornće et harmonique dans $\mathbb{D}$. On prouve ainsi que la fonction de Green associée à $\Delta \omega^{-1} \Delta$ - pour le problème de Dirichlet - est positive.

\section{Introduction}

If $\Delta$ stands for the Laplace operator in $\mathbb{R}^{n}(n=1,2,3, \ldots)$, we say that a real-valued function $u$ is harmonic on an open set if $\Delta u=0$ there, and subharmonic if $\Delta u \geq 0$. The maximum principle for subharmonic functions can be formulated as follows. Let $u$ be subharmonic in a domain $\Omega$ in $\mathbb{R}^{n}$, and let $D$ be a precompact subdomain. Suppose we have a function $v$, harmonic in $D$, such that $\left.u\right|_{\partial D} \leq\left. v\right|_{\partial D}$ (we can assume that both functions are continuous on the closure of $D$, so that we need not worry about boundary values). Then $u \leq v$ throughout $D$, with equality at some point in $D$ if and only if $u$ and $v$ coincide.

In 1908, Hadamard suggested that something similar may be true for the bilaplacian (also called the biharmonic operator) $\Delta^{2}$ on the plane $\mathbb{R}^{2}$, which we identify with $\mathbb{C}$, the complex plane. We say that a real-valued function $u$ on a domain $\Omega$ is biharmonic provided that $\Delta^{2} u=0$ there, and sub-biharmonic if $\Delta^{2} u \leq 0$ (one should think of $\Delta$ as a negative operator, which is the reason why the inequality is switched compared with the definition of subharmonic functions). At the time, it was known-more or less--that a variant can be formulated for circular disks. Let $\Omega$ be a planar domain, and $D$ a precompact circular disk in $\Omega$. We suppose that $u$ is a sub-biharmonic function on $\Omega$, and that $v$ is biharmonic on $D$. Then the following maximum principle holds:

$$
\left.u\right|_{\partial D} \leq\left. v\right|_{\partial D},\left.\quad \frac{\partial u}{\partial n}\right|_{\partial D} \leq\left.\left.\frac{\partial v}{\partial \mu}\right|_{\partial D} \Longrightarrow u\right|_{D} \leq\left. v\right|_{D},
$$


A maximum principle à la Hadamard for biharmonic operators...

where the normal derivative is calculated in the interior direction, and $u=v$ at some interior point if and only if $u$ and $v$ coincide identically. In the special case that the first inequality is an equality, then this maximum principle simply expresses the fact that the Green function for $\Delta^{2}$ on circular disks (with vanishing Dirichlet boundary data) is positive. When speaking of functions, the word positive is used to indicate that the function takes values in $[0,+\infty$ [. Hadamard, in his treatise on plaques élastiques encastrées [5], suggested that the Green function for $\Delta^{2}$ should be positive for a much larger collection of domains than the disks, and indicated that it appeared likely to be so for all convex regions with smooth boundary. This was later shown not to be the case, by Duffin, Lowner, and Garabedian. In fact, Garabedian [4] shows that it is false for an ellipse as soon as the ratio of the major axis to the minor axis exceeds $8 / 5$.

Here, we focus on a property exclusive to the circular disks:

$$
\int_{D} h(z) \mathrm{d} \Sigma(z)=r^{2} h\left(z_{0}\right)
$$

for all bounded harmonic functions on $D$, where $z_{0}$ is the center of the disk $D$, and $r$ is the radius. We let $\mathrm{d} \Sigma$ stand for the area measure, normalized by the factor $\pi^{-1}$, so that the unit disk $D$ gets area 1 . There is, however, a plethora of Riemann surfaces $R$ sheeted over some domain in the complex plane, with $R$ conformally equivalent to the unit disk (so the Riemann surface is the image of $\mathbb{D}$ under some holomorphic mapping $\mathbb{D} \rightarrow \mathbb{C}$ ), with the property that

$$
\int_{R} h(z) \mathrm{d} \Sigma(z)=r^{2} h\left(z_{0}\right)
$$

holds for all bounded harmonic functions $h$ on $R$, where $z_{0} \in R$ and $r$ is a positive parameter, and $\mathrm{d} \Sigma$ is lifted to each sheet of $R$. For instance, we obtain examples by considering the image of $\mathbb{D}$ under the mapping $z \mapsto \alpha z^{N}$, where $\alpha$ is some positive constant, and $N=1,2,3, \ldots$ It is a consequence of our main result (formulated below) that the Green function for $\Delta^{2}$ is positive on all such surfaces $R$ (even when the surface has rough boundary, it is possible to make sense of the boundary conditions). That is, if we replace "convex" by "centered" (interpreted in the above sense as a mean value property for harmonic functions), then Hadamard was right after all! Let $\Gamma_{R}(z, \zeta)$ denote the biharmonic Green function on $R$. Applying Green's formula twice shows that the positivity of $I_{R}$ is equivalent to the following maximum principle, with $u$ sub-biharmonic and $v$ biharmonic:

$$
\left.u\right|_{\partial R}=\left.\eta\right|_{\partial R} \text { and }\left.\frac{\partial u}{\partial n}\right|_{\partial R} \leq\left.\left.\frac{\partial v}{\partial n}\right|_{\partial R} \Longrightarrow u\right|_{R} \leq\left. v\right|_{R} .
$$

Let $\phi: \mathbb{D} \rightarrow R$ be the conformal map which takes the origin onto the center point $z_{0}$ of $R$. We may choose to view $\phi$ as a holomorphic functions with values in $\mathbb{C}$, by composing the conformal map $\mathrm{D} \rightarrow R$ with the projection from the Riemann surface to the domain that it is sheeted over. It is well known that $\left|\phi^{\prime}\right|^{-2} \Delta(u \circ \phi)=(\Delta u) \circ \phi$, and it follows from this formula that $\Gamma_{R}(\phi(z), \phi(\zeta))$ is the Green function on the unit disk for the weighted biharmonic operator $\Delta\left|\phi^{\prime}\right|^{-2} \Delta$, denoted by $\Gamma_{\left|\phi^{\prime}\right|^{2}}$. The reproducing property for $R$, Equation (1.1), becomes

$$
\int_{\mathbb{D}} h(z)\left|\phi^{\prime}(z)\right|^{2} \mathrm{~d} \Sigma(z)=r^{2} h(0)
$$

for all bounded harmonic functions $h$ on $\mathbb{D}$. 


\section{H. Hedenmalm et al.}

\section{Main result}

An area-summable function $\omega: \mathbb{D} \rightarrow[0,+\infty[$ is said to be a weight; it is logarithmically subharmonic if $\log \omega$ is subharmonic on $\mathbb{D}$, and reproducing if

$$
\int_{D} h(z) \omega(z) \mathrm{d} \Sigma(z)=h(0)
$$

holds for all bounded harmonic functions $h$ on $\mathbb{D}$. In the context of (1.2), the function $\omega=r^{-2}\left|\phi^{\prime}\right|^{2}$ is of this type. Our main result is the following:

THEOREM 2.1. - Let $\omega$ be a logarithmically subharmonic reproducing weight on $\mathbf{D}$. Then the Green function $\Gamma_{\omega}$ for the weighted biharmonic operator $\Delta \omega^{-1} \Delta$-with respect to the Dirichlet problem-is positive throughout $\mathbb{D} \times \mathbb{D}$.

A particular consequence is that the biharmonic Green function $\Gamma_{R}$ on the Riemann surface $R$ mentioned previously is positive. Let us translate Theorem 2.1 into the form of a maximum principle. We say that a function $u$ defined on a domain is $\omega$-biharmonic provided that $\Delta \omega^{-1} \Delta u=0$ there, and $s u b-\omega$-biharmonic if $\Delta \omega^{-1} \Delta u \leq 0$. Suppose $u$ is sub- $\omega$-biharmonic and $v$ is $\omega$-biharmonic. The positivity of the Green function then leads to the maximum principle:

$$
\left.u\right|_{T}=\left.v\right|_{\mathbb{T}} \text { and }\left.\frac{\partial u}{\partial \mathrm{n}}\right|_{\mathrm{T}} \leq\left.\left.\frac{\partial v}{\partial \mathrm{n}}\right|_{\mathrm{T}} \Longrightarrow u\right|_{\mathrm{D}} \leq\left. v\right|_{\mathbb{D}}
$$

at least if $u$ and $v$ are sufficiently smooth.

\section{Applications to the Bergman spaces}

For $p$ with $0<p<+\infty$, the Bergman space $A^{p}(\mathbb{D})$ consists of all holomorphic functions $f: \mathbb{D} \rightarrow \mathbb{C}$ with bounded norm

$$
\|f\|_{A^{p}}-\left(\int_{D}|f(z)|^{p} \mathrm{~d} \Sigma(z)\right)^{\frac{1}{p}}<+\infty .
$$

It is a Hilbert space for $p=2$, a Banach space for $1 \leq p<+\infty$, and a quasi-Banach space for $0<p<1$. An inner function in $A^{p}(\mathbb{D})$ is a function $\varphi \in A^{p}(\mathbb{D})$ with the property that

$$
\int_{\mathbf{D}} h(z)|\varphi(z)|^{p} \mathrm{~d} \Sigma(z)=h(0)
$$

holds for all bounded harmonic functions $h$ on $\mathbb{D}$. The function $|\varphi|^{p}$ is logarithmically subharmonic, and (3.1) expresses that this weight should be reproducing for 0 . For $p=2$, the function $\varphi=r^{-1} \phi^{\prime}$ in (1.2) is of this type. The inner functions of $A^{p}(\mathbb{D})$ have been studied rather extensively in reccnt years, primarily because of their use for the factorization of functions with respect to zeros and their relevance for operator theory (see [7], [2], [3], and [1]). They are analogous to the classical inner functions which play a central role in the function theory of the Hardy spaces $\mathrm{H}^{p}(\mathbb{D})$, for $0<p<+\infty$. $\Lambda s$ we apply our main result to the Bergman spaces $A^{p}(\mathbb{D})$, we arrive at the following. Given a zero sequence $A$ in $\mathbb{D}$ for the space $A^{p}(\mathbb{D})$, let $M_{A}$ be the subspace of all functions in $A^{p}(\mathbb{D})$ that vanish at all points of $A$, with multiplicities as prescribed by the sequence. Subspaces of the type $M_{A}$ are referred to as zero-set subspaces. Let $\varphi_{A}$ be the function that maximizes $|\varphi(0)|$, given that $\varphi$ vanishes on $A$ and has norm 1 (this does not define $\varphi_{A}$ uniquely, because we can always multiply by 
A maximum principle à la Hadamard for biharmonic operators...

a unimodular constant, but this is the only obstruction; if $A$ contains the origin, we need to maximize the first non-vanishing derivative at the origin). The function $\varphi_{A}$ is an inner function in $A^{p}(\mathbb{D})$, and it has no extraneous zeros; in fact, it generates $M_{A}$ as an invariant subspace [1] (see below for a definition of the term invariant subspace). Duren, Khavinson, Shapiro, and Sundberg coined the term canonical zero divisors for these functions $\varphi_{A}$. For two zero sequences $A$ and $B$, such that $A$ is contained in $B$, it follows from Theorem 2.1 that

$$
\left\|\varphi_{A} f\right\|_{A^{p}} \leq\left\|\varphi_{B} f\right\|_{A^{p}}
$$

for all holomorphic functions $f$ on $\mathbb{D}$. This means that the canonical zero divisors are monotonic with respect to Korenblum domination along the lattice of zero-set subspaces. Following Korenblum [10], we say that given two function $F$ and $G$ in $A^{p}(\mathbb{D}), G$ dominates $F$, written $F \prec G$, provided $\|F q\|_{A^{p}} \leq\|G q\|_{A^{p}}$ holds for all polynomials $q$. The relation $\varphi_{A} \prec \varphi_{B}$ for $A \subset B$ was conjectured by Hedenmalm in [8], [9] as well as in Problem 12.13 in the Havin-Nikolski problem hook [6]. A consequence of this result is the following. We say that a closed subspace $M$ of $A^{p}(\mathbb{D})$ is invariant provided that $S f \in M$ whenever $f \in M$, where $S$ is the shift operator: $S f(z)=z f(z)$. A particularly simple collection are the zero-set subspaces, as described above. These have the property that they have index 1 (with the exception of the trivial invariant subspace $\{0\}$ ), meaning that the dimension of $M / S M$ is 1 . If an invariant subspace $M$ with index 1 contains a zero-set subspace, then $M$ itself is a zero set subspace.

\section{Method of proof}

A few words should be said about the proof of the main result, the positivity of the Green function $\Gamma_{\omega}$. An application of the laplacian with respect to both coordinates leads to the identity

$$
\Delta_{z} \Delta_{\zeta} \Gamma_{\omega}(z, \zeta)=\omega(z) \delta_{\zeta}(z)-\omega(z) \omega(\zeta) Q_{\omega}(z, \zeta), \quad(z, \zeta) \in \mathbb{D} \times \mathbb{D}
$$

where $\Delta_{\zeta}$ is the Dirac point mass at $\zeta$, and $Q_{\omega}$ stands for the reproducing kernel of the space $\mathrm{HP}^{2}(\mathbb{D}, \omega)$ obtained as the closure of the harmonic polynomials with respect to the norm

$$
\|f\|_{\omega}=\left(\int_{D}|f(z)|^{2} \omega(z) \mathrm{d} \Sigma(z)\right)^{\frac{1}{2}}
$$

A necessary condition for $\Gamma_{\omega}$ to be positive is that $Q_{\omega}$ is negative away from the diagonal on $\mathbb{T} \times \mathbb{T}$. We have found the following reverse implication. We first prove that off the diagonal,

$$
Q_{\omega}(z, \zeta) \leq-\left(\frac{1}{\omega(z)}+\frac{1}{\omega(\zeta)}\right) \frac{1}{|z-\zeta|^{2}}, \quad(z, \zeta) \in \mathbb{T} \times \mathbb{T},
$$

and second, that this implies the positivity of $\Gamma_{\omega}$ by a successive integration along the weighted Hele-Shaw flow to be described.

Let $\Omega$ be a smoothly bordered finitely connected bounded domain in $\mathbb{C}$, and suppose $\omega: \Omega \rightarrow$ $[0,+\infty[$ is a logarithmically subharmonic. For simplicity we consider only weights $\omega$ that are real analytic up to the boundary and have $0<\omega(z)$ at all points of $\bar{\Omega}$. For a point $z_{0} \in \Omega$ and a radial parameter $r, 0<r<+\infty$, we consider precompact subdomains $D$ (generalized disks centered at $z_{0}$ with radius $r$ ) with the reproducing property

$$
\int_{D} h(z) \omega(z) \mathrm{d} \Sigma(z)=r^{2} h\left(z_{0}\right)
$$




\section{H. Hedenmalm et al.}

for all bounded harmonic functions $h$ on $D$. We add the requirement that there be an inequality $\leq$ in (4.2) when $h$ is bounded and subharmonic on $D$. Then there is at most one Hele-Shaw flow domain $D=D(r)$ (up to sets of zero area), and when they exist, the domains grow with the parameter $r$. There exists a critical "radius" value $\rho\left(z_{0}\right)$ at which the boundary of the $\omega$-disk touches $\partial \Omega$ for the first time, and below that value, the generalized disks are well-defined, and above, they simply do not exist. Assuming that the underlying domain $\Omega$ is simply connected, each $\omega$-disk $D(r)$ is simply connected, and its boundary is a regular real analytic closed curve. Also, the domains $D(r)$ depend on $r$ in a real analytic fashion. The proof of these statements is based on Sakai's classification of free boundaries [11].

We wish to point out that the above results have been obtained in dimension $n=2$ only. It is not clear what the appropriate generalization to higher dimensional $\mathbb{R}^{n}$, for $n=3,4,5, \ldots$, should look like. These problems deserve further investigation.

The details of this paper will appear elsewhere.

\section{References}

[1] Aleman A., Richter S., Sundberg C., Beurling's theorem for the Bergman space, Acta Math. 177 (1996) 275-310

12] Duren P.L., Khavinson D., Shapiro H.S., Sundberg C., Contractive zero-divisors in Bergman spaces, Pacific J. Math. 157 (1993) $37-56$.

[3] Duren P.L., Khavinson D., Shapiro H.S., Sundberg C., Invariant subspaces and the biharmonic equation, Michigan Math. J. 41 (1994) 247-259.

|4| Garabedian P.R., A partial differential equation arising in conformal mapping, Pacific J. Math. 1 (1951) 485-524

(5) Hadamard J., Guvres de Jacques Hadamard, Vols. 1-4, Editions du Centre national de la recherche scientifique, Paris, 1968.

[6] Havin V.P.. Nikolski N.K. (Eds.), Linear and complex analysis. Problem book 3, Part II. Lect. Notes in Math. 1574. Springer-Verlag, Berlin. 1994.

[7] Hedenmalm H., A factorization theorem for square area-integrable analytic functions, J. Reine Angew. Math. 421 (1991) $45-68$

[8] Hedenmalm $\mathrm{H}$., A computation of the Green function for the weighted biharmonic operators $\Delta|z|^{-2 n} \Delta$, with $\gamma>-1$, Duke Math. 1. 75 (1994) $51-78$

19] Hedenmalm H., Open problems in the function theory of the Bergman space, Festschrift in honour of Lennart Carleson and Yngve Domar (Uppsala, 1993), Acta Univ. Upsaliensis Skr. Uppsala Univ. C Organ. Hist., 58, Uppsala Univ.. Uppsala, 1995. pp. $153 \cdots 169$.

[10| Korenblum B. A maximum principle for the Bergman space, Publ. Mat. 35 (1991) 479-486.

111] Sakili M.. Regularity of a boundary having a Schwarz function, Acta Math. 166 (1991) 263297. 